\title{
Heart Failure Treated with Low-dose Milrinone in a Full-term Newborn
}

\author{
Šebková S. ${ }^{1}$, Tomek V. ${ }^{2}$, Zemanová P. ${ }^{1}$, Janota J..$^{1,3}$ \\ ${ }^{1}$ Department of Neonatology, Thomayer Hospital, Prague, Czech Republic; \\ ${ }^{2}$ Kardiocentrum and Cardiovascular Research Centre, University Hospital Motol, \\ Prague, Czech Republic; \\ ${ }^{3}$ Institute of Pathological Physiology, First Faculty of Medicine, Charles University in \\ Prague, Prague, Czech Republic
}

Received September 23, 2011; Accepted January 16, 2012.

Key words: Milrinone - Newborn - Heart failure - Pulmonary hypertension

Abstract: A term newborn with a hypocontractile myocardium complicating persistent pulmonary hypertension of the newborn was successfully treated with a low-dose phosphodiesterase III inhibitor milrinone. Echocardiography diagnosed heart failure with a left ventricular ejection fraction of $35 \%$ and a left ventricular shortening fraction of $18 \%$ and severe persistent pulmonary hypertension of the newborn with oxygenation index of 28. Milrinone was started at an initial dose of $50 \mathrm{mcg} / \mathrm{kg}$, followed by continuous infusion of $0.20 \mathrm{mcg} / \mathrm{kg} / \mathrm{min}$. With lowdose milrinone oxygenation index decreased to 3 within 6 hours, left ventricular ejection fraction and left ventricular shortening fraction increased to $57 \%$, and $30 \%$, respectively. Low doses of milrinone might be promising in the treatment of heart failure and persistent pulmonary hypertension of the newborn in term newborns.

Mailing Address: Assoc. Prof. Jan Janota, MD., PhD., Department of Neonatology, Thomayer Hospital, Vídeňská 800, 14059 Prague 4, Czech Republic; Mobile Phone: +420 603587 632; Fax: +420 261083 740; e-mail: jan.janota@ftn.cz 


\section{Introduction}

Milrinone is an effective lusitropic agent augmenting ventricular diastolic relaxation in addition to systolic contractility. Its cellular actions include inhibition of phosphodiesterase-mediated degradation of cyclic adenosine monophosphate, increased activation of protein kinase A, phosphorylation of phospholamban, and increased intracellular calcium (Rapundalo et al., 1986). This results in an increase in cardiac output while reducing pulmonary capillary wedge pressure and systemic vascular resistance with minimal increase in myocardial oxygen demand (McMahon et al., 2006). Because of its pulmonary vasodilator properties and cardiotropic effects, milrinone is a promising therapy for neonates with persistent pulmonary hypertension of the newborn (Tanaka et al., 1991; Bassler et al., 2006; McNamara et al., 2006). The protocol for dosage of milrinone in newborns has not been established yet (Bassler et al., 2010; Sehgal, 2011). High-dose milrinone $(\geq 0.25 \mathrm{mcg} / \mathrm{kg} / \mathrm{min})$ is currently reported to be used in term and preterm newborns (Danhaive et al., 2005; Paradisis et al., 2009; Bassler et al., 2010; Tzialla et al., 2010). Low-dose milrinone ( $<0.25 \mathrm{mcg} / \mathrm{kg} / \mathrm{min})$ was shown to be as effective as high dose in preterm newborns (Paradisis et al., 2007). Low-dose milrinone for the treatment of cardiac failure and persistent pulmonary hypertension of the newborn might be effective in term newborns as well (Hoffman et al., 2003; Bassler et al., 2010). Successful treatment of cardiac failure and persistent pulmonary hypertension with low-dose milrinone in term newborn without congenital heart defect has not been described yet.

\section{Case report}

The patient, a female infant weighing 3.16 kilograms, was born in a primary health centre at 38 weeks gestation following a precipitous delivery complicated by foetal bradycardia. The Apgar scores at both 1 and 5 minutes were 9 . Subsequently, within 30 minutes of life she developed dyspnea and cyanosis, and required inhaled oxygen (fraction of inspired oxygen up to 0.3 , postductal oxygen saturation $88 \%$ ). She was intubated, and transported to our hospital at 9 hours of age.

On admission, the infant presented with severe persistent pulmonary hypertension of the newborn confirmed by echocardiography - tricuspid regurgitation third degree with peak velocity $3.4 \mathrm{~m} / \mathrm{s}$ and right ventricle (RV) peak pressure of $53 \mathrm{~mm} \mathrm{Hg}$, right-to-left ductal flow, left ventricular ejection fraction (EF) 63\%, left ventricular shortening fraction (SF) 32\%, diameter of the left ventricle (LVD) $17.2 \mathrm{~mm}$. The infant was ventilated on assist control+volume guarantee requiring peak inspiratory pressure of $25 \mathrm{~cm} \mathrm{H}_{2} \mathrm{O}$ and fraction of inspired oxygen up to 1.0. Postductal oxygen saturation was $88 \%$ and the oxygenation index $(\mathrm{OI})$ was 28. Invasive blood pressure was 66/27 mm Hg (mean 43 mm Hg) (Figures 1 and 2).

Treatment was commenced which included diuretics, sodium bicarbonate, boluses of normal saline, fresh frozen plasma, muscle relaxants, sedation, and antibiotics. High dose dopamine was administered two hours after admission for 


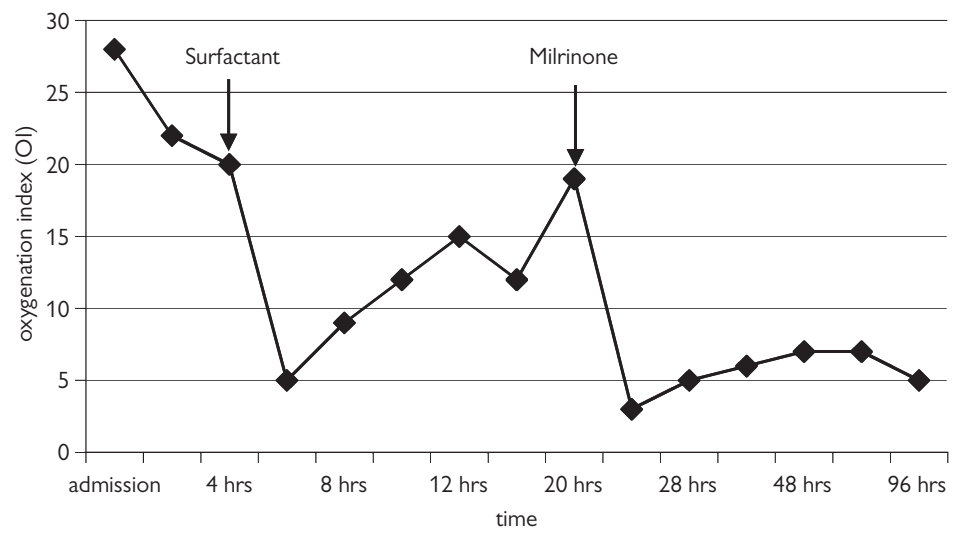

Figure 1 - Changes in oxygenation index in a patient with persistent pulmonary hypertension of the newborn and circulatory failure. Effect of treatment with surfactant and milrinone on oxygenation index.

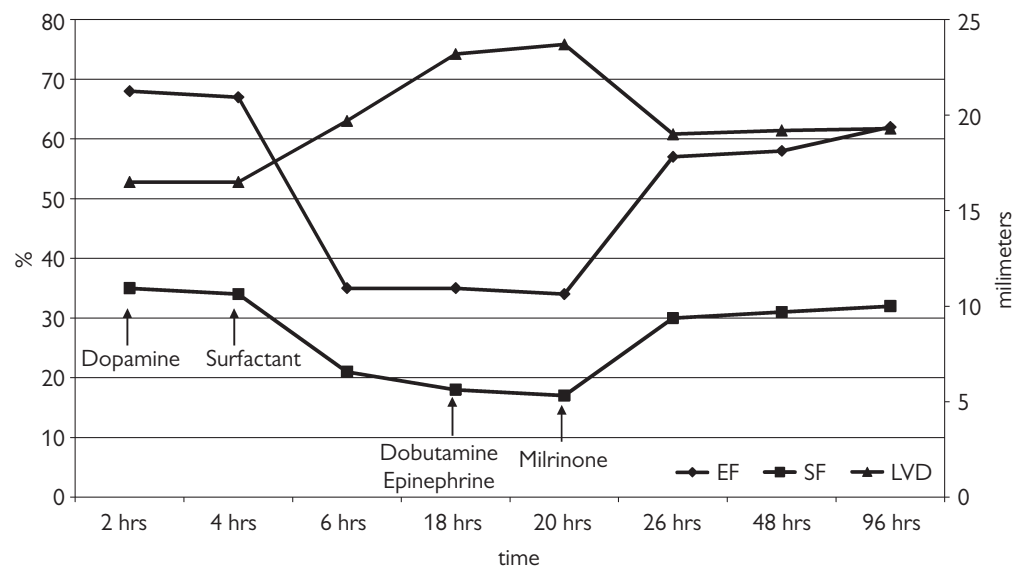

Figure 2 - Changes in ejection fraction (EF), shortening fraction (SF), and diameter of the left ventricle (LVD) in a patient with persistent pulmonary hypertension of the newborn and circulatory failure.

Effect of treatment with surfactant and milrinone on function and diameter of the left ventricle.

treatment of systemic hypotension during pulmonary hypertension. The newborn was assigned to nitric oxide treatment, but according to institutional protocol and because of progression of persistent pulmonary hypertension of the newborn (PPHN) - tricuspid regurgitation, third degree with RV peak pressure $60 \mathrm{~mm} \mathrm{Hg}$, right atrium dilatation, EF 68\%, SF 35\%, LVD $16.5 \mathrm{~mm}$ received surfactant before nitric oxide administration (4 hours after admission) (Kinsella, 2006) (Figures 1 and 2). Institutional indication criteria for $\mathrm{NO}$ administration are: full-term or latepreterm newborns, hypoxic respiratory failure with oxygenation index 25 and more, administration in first week of life, echocardiography confirmed right to left

Šebková S.; Tomek V.; Zemanová P.; Janota J. 
extrapulmonary shunts and optimizing ventilatory support including administration of surfactant (Alpan, 2009). For evaluation of severity of the pulmonary illness, oxygenation index $(\mathrm{Ol})$ was used. High $\mathrm{Ol}$ is objective measure of the degree of respiratory failure. OI above 25 indicates severe respiratory failure, OI 33-40 indicates that extracorporeal membrane oxygenation should be considered (Bayrakci et al., 2007; Betit and Craig, 2009). The equation of Ol is: $\mathrm{OI}=\mathrm{F}_{\mathrm{i}} \mathrm{O}_{2} \times$ mean airway pressure $\times 100 / \mathrm{P}_{\mathrm{a}} \mathrm{O}_{2}$ where: $\mathrm{F}_{\mathrm{i}} \mathrm{O}_{2}$ - inspired oxygen concentration, $\mathrm{P}_{\mathrm{a}} \mathrm{O}_{2}$ - partial pressure of arterial $\mathrm{O}_{2}$.

After surfactant administration, an improvement in oxygenation was observed within 2 hours. Oxygenation index decreased from 22 to 5 (Figure 1). However, despite the improvement in persistent pulmonary hypertension of the newborn (tricuspid regurgitation first degree with RV peak pressure $32 \mathrm{~mm} \mathrm{Hg}$ ), the patient developed acute heart failure with a hypocontractile myocardium, reduced $\mathrm{EF}$ of $35 \%$, and reduced SF of 21\%, LVD $19.7 \mathrm{~mm}$ and mitral regurgitation of first to second degree. For altered heart contractility high doses of dobutamine and epinephrine were added to the existing therapy (18 hours after admission) (Figure 2). Echocardiography revealed myocardium with low contractility, dilatation of the left ventricle with LVD $23.2 \mathrm{~mm}$, EF 35\%, and SF 18\%. Oxygenation index increased to 19. Milrinone was started at an initial dose of $50 \mathrm{mcg} / \mathrm{kg}$, followed by continuous infusion of $0.20 \mathrm{mcg} / \mathrm{kg} / \mathrm{min}$ (Paradisis et al., 2007). One hour after administration of milrinone echocardiography confirmed improved contractility of myocardium with EF 50\%, SF 23\%, LVD $22.1 \mathrm{~mm}$, and mitral regurgitation first degree and RV peak pressure of $26 \mathrm{~mm} \mathrm{Hg.} 6$ hours after administration of milrinone, we documented significant improvement of oxygenation $(\mathrm{OI}=3)$. Echocardiography showed an improvement in myocardial contractility with normalization of left ventricular ejection fraction (EF 57\%), significant improvement in left ventricular shortening fraction (SF 30\%), and reduction of diameter of the left ventricle (LVD $19 \mathrm{~mm}$ ) with mitral regurgitation first degree (Figures 1 and 2 ).

Milrinone was stopped on day 8 , inotropes discontinued on day 12 . The newborn was successfully extubated on day 13 . Patient was discharged home on day 29 of life. Follow-up at 6 and 12 months of age revealed normal findings on physical and neurological examination. Growth and development were within normal limits.

\section{Discussion}

Persistent pulmonary hypertension of the newborn is present when an infant with echocardiographically confirmed structurally normal heart has severe hypoxemia, with a partial pressure of arterial oxygen less than 5-6 kPa in fraction of inspired oxygen of 1.0 and/or there is an evidence of right-to-left ductal shunt (Greenough and Milner, 2005). The incidence of persistent pulmonary hypertension of the newborn is $0.43-6.8 / 1.000$ live births and the mortality is $10-20 \%$. Survivors have high morbidity, neurodevelopmental and audiological impairment, cognitive delays, hearing loss, and a high rate of rehospitalisation (Greenough and Milner, 2005). 
Pulmonary hypertension of the newborn may be result of underdevelopment of the lung together with its vascular bed (congenital diaphragmatic hernia), maladaptation of the pulmonary bed to the transition occurring around the time of birth (perinatal stress, haemorrhage, aspiration, hypoxia, hypoglycaemia), and maldevelopment of the pulmonary vascular bed in utero from known or unknown cause. However, the clinical manifestations of PPHN are often not attributable to a single physiologic or structural entity, and many disorders exhibit more than one underlying pathology. Often, even when there is evidence of perinatal or postnatal stress, the underlying cause of PPHN had been secondary to an in utero process of some duration (Alpan, 2009). Our patient was born after precipitous delivery complicated by foetal bradycardia. The Apgar scores at 1 and 5 minutes were 9 . Umbilical cord $\mathrm{pH}$ was not recorded. We could hypothesize that the patient might suffer from hypoxia before labour resulting in PPHN. There were no significant signs of perinatal stress, hypoxia, or hypoglycaemia. The patient was diagnosed with idiopathic PPHN.

The optimal approach to the management of persistent pulmonary hypertension of the newborn remains controversial. Inhaled nitric oxide is currently regarded as the gold standard therapy, but with as many as $30 \%$ of cases failing to respond, has not proven to be the single magic bullet (Greenough and Milner, 2005). Milrinone is a promising therapy for many neonates with persistent pulmonary hypertension of the newborn because of its preferential pulmonary vasodilator properties (Tanaka et al., 1991) and cardiotropic effects (Rapundalo et al., 1986; McNamara et al., 2006). This drug is also used in paediatric cardiac intensive care units to improve myocardial contractility and reduce afterload.

Cellular actions of milrinone include inhibition of phosphodiesterase-mediated degradation of cyclic adenosine monophosphate (CAMP). The role of cAMP in the pulmonary vasculature during the transition from intrauterine to extrauterine life is poorly understood. Animal studies suggest that phosphodiesterase 3 expression and activity in pulmonary arteries dramatically increase after birth, with concomitant decrease in steady-state cAMP. Therefore the vasodilatatory effects of milrinone might be more pronounced in newborns with PPHN (Chen et al., 2009). The neonatal myocardium may be more dependent on calcium for inotropy than the adult myocardium. There is less intracellular calcium, and greater dependence on trans-sarcolemmal calcium flux (Hsu and Pearson, 2009). Phosphodiesterase inhibitors as milrinone affect myocardial function in these three ways: (A) increased inotropy as a result of CAMP - mediated increase in transsarcolemmal calcium influx, (B) peripheral vasodilatation secondary to removal of free intracellular calcium by causing its uptake by sarcoplasmic reticulum and (C) myocyte relaxation possibly due to improved actin-myosin complex dissociation (Sehgal et al., 2011).

In this case-report we describe our experience with the effect of milrinone on heart contractility and persistent pulmonary hypertension of the newborn in 
term newborn. After surfactant administration, there was a significant decrease in PPHN. In the same time there was a significant decrease in cardiac contractility and worsening of ventilation-perfusion mismatch followed by increase of OI (Bengur and Meliones, 1998; Stayer and Liu, 2010). Administration of milrinone was followed by improvement in cardiac contractility and further decrease of PPHN (RV peak pressure bellow $30 \mathrm{~mm} \mathrm{Hg}$ ). We conclude that possible mechanism how milrinone influenced the patient was improved cardiac contractility and subsequent normalization of cardiac function. PPHN decreased after surfactant and normalized during milrinone therapy.

The protocol for administration of milrinone in newborns is not established. High-dose milrinone is effective in term and preterm newborns with low cardiac output and PPHN. Low-dose milrinone was shown to be as effective as high dose with possibly less adverse effects (Bassler et al., 2010). There are no randomized clinical studies comparing high and low doses of milrinone available. This is the reason why milrinone is currently not widely used in neonatology. The only on-going randomized trial comparing low and high doses of milrinone in newborns is reported in Cochrane Database of Systematic Reviews (Bassler et al., 2010). No results of this study have been published yet.

Following adverse effects of milrinone are currently described in newborn: hypokalaemia, thrombocytopenia, abnormal liver function tests, ventricular arrhythmias and hypotension (Anderson, 2009). Hypotension, renal failure (Zuppa et al., 2006) and intraventricular haemorrhage (Bassler et al., 2006) were reported in term infants during high-dose milrinone administration. We didn't observe any of the above mentioned adverse reactions during milrinone therapy in our patient.

The significant improvement of heart contractility we reached with low doses of milrinone. Initial low doses of milrinone were chosen because of illness severity and the potential risk of adverse effects, mainly profound hypotension and bleeding (Bassler et al., 2006; McNamara et al., 2006). With low doses, we observed significant improvement in myocardial contractility within 6 hours; therefore it was not necessary to increase the dose. Low-dose milrinone was successfully used in term newborns with congenital heart defects and low cardiac output (Hoffman et al., 2003; Zuppa et al., 2006). There is no report of low-dose milrinone used in term newborns with PPHN without congenital heart defect. In our case report we were able to show for the first time the successful use of low-dose milrinone in PPHN and cardiac failure in term newborn without congenital heart defect.

\section{Conclusion}

This case-report shows, that the low doses of phosphodiesterase 3 inhibitor milrinone might be successfully used for treatment of acute heart failure with a hypocontractile myocardium as a complication of persistent pulmonary hypertension of the newborn. 


\section{References}

Alpan, G. (2009) Persistent pulmonary hypertension of the newborn. In: Neonatology: Management, Procedures, On-Call Problems, Diseases and Drugs, $6^{\text {th }}$ Edition. Gomella, T. L., Cunningham, M. D., Eyal, F. G., Tuttle, D., pp. 636-644, McGraw-Hill, New York.

Anderson, A. L. (2009) Commonly used medications. In: Neonatology: Management, Procedures, On-Call Problems, Diseases and Drugs, $6^{\text {th }}$ Edition. Gomella, T. L., Cunningham, M. D., Eyal, F. G., Tuttle, D., Pp. 729-787, McGraw-Hill, New York.

Bassler, D., Choong, K., McNamara, P., Kirpalani, H. (2006) Neonatal persistent pulmonary hypertension treated with milrinone: four case reports. Biol. Neonate 89(1), 1-5.

Bassler, D., Kreutzer, K., McNamara, P., Kirpalani, H. (2010) Milrinone for persistent pulmonary hypertension of the newborn. Cochrane Database Syst. Rev. 10(11), CD007802.

Bayrakci, B., Josephson, C., Fackler, J. (2007) Oxygenation index for extracorporeal membrane oxygenation: is there predictive significance? J. Artif. Organs 10(1), 6-9.

Bengur, A. R., Meliones, J. N. (1998) Cardiogenic shock. New Horiz. 6(2), 139-149.

Betit, P., Craig, N. (2009) Extracorporeal membrane oxygenation for neonatal respiratory failure. Respir. Care 54(9), 1244-1251.

Chen, B., Lakshminrusimha, S., Czech, L., Groh, B. S., Gugino, S. F., Russel, J. A. (2009) Regulation of phosphodiesterase 3 in the pulmonary arteries during the perinatal period in sheep. Pediatr. Res. 66(6), 682-687.

Danhaive, O., Margossian, R., Geva, T., Kourembanas, S. (2005) Pulmonary hypertension and right ventricular dysfunction in growth-restricted, extremely low birth weight neonates. J. Perinatol. 25, 495-499.

Greenough, A., Milner, A. D. (2005) Pulmonary disease of the newborn: Acute respiratory disease. In: Roberton's Textbook of Neonatology, $4^{\text {th }}$ Edition. Rennie, J. M., Elsevier, London.

Hoffman, T. M., Wernovsky, G., Atz, A. M., Kulik, T. J., Nelson, D. P., Chang, A. C., Bailey, J. M., Akbary, A., Kocsis, J. F., Kaczmarek, R., Spray, T. L., Wessel, D. L. (2003) Efficacy and safety of milrinone in preventing low cardiac output syndrome in infants and children after corrective surgery for congenital heart disease. Circulation 107(7), 996-1002.

Hsu, D. T., Pearson, G. D. (2009) Heart failure in children: part I: history, etiology, and pathophysiology. Circ. Heart Fail. 2(1), 63-70.

Kinsella, J. P. (2006) Inhaled nitric oxide therapy. In: Manual of Neonatal Respiratory Care, $2^{\text {nd }}$ Edition. Donn, S. M., Sinha, S. K., Pp. 426-427, Mosby, Philadelphia.

McMahon, C. J., Murchan, H., Prendiville, T., Burch, M. (2006) Long-term support with milrinone prior to cardiac transplantation in neonate with left ventricular noncompaction cardiomyopathy. Pediatr. Cardiol. 28(4), 317-318.

McNamara, P., Laique, F., Muang, S., Whyte, H. (2006) Milrinone improves oxygenation in neonates with severe persistent pulmonary hypertension of the newborn. J. Crit. Care 21(2), 217-222.

Paradisis, M., Jiang, X., McLachlan, A. J., Evans, N., Kluckow, M., Osborn, D. (2007) Population pharmacokinetics and dosing regimen design of milrinone in preterm infants. Arch. Dis. Child. Fetal Neonatal Ed. 92(3), F204-F209.

Paradisis, M., Evans, N., Kluckow, M., Osborn, D. (2009) Randomized trial of milrinone versus placebo for prevention of low systemic blood flow in very preterm infants. J. Pediatr. 154(2), 189-195.

Rapundalo, S. T., Grupp, I., Grupp, G., Matlib, M. A., Solaro, R. J., Schwartz, A. (1986) Myocardial actions of milrinone: characterization of its mechanism of action. Circulation 73(3), 134-144.

Sehgal, A. (2011) Haemodynamically unstable preterm infant: an unresolved management conundrum. Eur. J. Pediatr. 170(10), 1237-1245.

Šebková S.; Tomek V.; Zemanová P.; Janota J. 
Sehgal, A., Francis, J. V., Lewis, A. I. (2011) Use of milrinone in the management of haemodynamic instability following duct ligation. Eur. J. Pediatr. 170(10), 115-119.

Stayer, S. A., Liu, Y. (2010) Pulmonary hypertension of the newborn. Best Pract. Res. Clin. Anaesthesiol. 24(3), 375-386.

Tanaka, H., Tajimi, K., Moritsune, O., Kobayashi, K., Okada, K. (1991) Effects of milrinone on pulmonary vasculature in normal dogs and in dogs with pulmonary hypertension. Crit. Care Med. 19(1), 68-74.

Tzialla, C., Cerbo, R. M., Perotti, G., Stronati, M. (2010) Persistent pulmonary hypertension of the newborn refractory to inhaled nitric oxide-treated with milrinone: a case report. Turk. J. Pediatr. 52(1), 78-80.

Zuppa, A. F., Nicolson, S. C., Adamson, P. C., Wernovsky, G., Mondick, J. T., Burnham, N., Hoffman, T. M., Gaynor, J. W., Davis, L. A., Greeley, W. J., Spray, T. L., Barrett, J. S. (2006) Population pharmacokinetics of milrinone in neonates with hypoplastic left heart syndrome undergoing stage I reconstruction. Anesth. Analg. 102(4), 1062-1069. 